\title{
On nonexpansive and accretive operators in Banach spaces
}

\section{Dongfeng Li}

School of Information Engineering, North China University of Water Resources and Electric Power, Zhengzhou 450011, China.

\author{
Communicated by Y. J. Cho
}

\begin{abstract}
The purpose of this article is to investigate common solutions of a zero point problem of a accretive operator and a fixed point problem of a nonexpansive mapping via a viscosity approximation method involving a $\tau$-contractive mapping. (C)2017 All rights reserved.
\end{abstract}

Keywords: Accretive operator, approximation solution, viscosity method, variational inequality.

2010 MSC: 47H05, 65J15.

\section{Introduction}

Accretive and monotone operator equations have been one of the most active research areas of optimization theory and nonlinear functional analysis. As it is well-known, zero point theorems of accretive and monotone operator can be deduced from existence theorems for differential equations, see, e.g. $[2,9,20,22]$ and the references therein. One of efficient methods to solve the accretive and monotone operator equations is the iterative method.

There are several significant classes of accretive and monotone operators which enjoy remarkable properties not shared by all such operators. We refer, for example, to strong monotone operators, $\mathrm{m}$ accretive operators, maximal monotone operators and inverse-strongly accretive operators, see [4, 10, 11, 17] and the references therein. In particular, m-accretive operators are of utmost importance in nonlinear functional analysis and optimization theory, see $[1,20,23,24]$ and the references therein. It is known that every m-accretive operator, in the framework of Hilbert spaces, is maximal monotone. Let $\mathrm{H}$ be a real Hilbert space with inner product $\langle x, y\rangle$ and induced norm $\|x\|=\sqrt{\langle x, x\rangle}$ for $x, y \in H$. Let $C$ be a nonempty closed and convex subset of $\mathrm{H}$. One known example of maximal mapping is $\partial f$, the subdifferential of a convex proper closed function $\mathrm{f}: \mathrm{H} \rightarrow \Omega$, where $\Omega:=(-\infty, \infty]$, which is defined by

$$
\partial f(x):=\left\{x^{*} \in H: f(x)+\left\langle y-x, x^{*}\right\rangle \leqslant f(y), \forall y \in H\right\}, \quad \forall x \in H .
$$

Rockafellar [21] proved that $\partial f$ is a maximal monotone operator. It is easy to verify that $0 \in \partial f(v)$ if and only if $f(v)=\min _{x \in H} f(x)$. Another example is $M+N_{C}$, where $M$ is a single-valued maximal monotone 
mapping that is continuous on $\mathrm{C}$, and $\mathrm{N}_{\mathrm{C}}$ is the normal cone mapping

$$
\mathrm{N}_{\mathrm{C}}(\mathrm{x}):=\left\{\mathrm{x}^{*} \in \mathrm{H}:\left\langle\mathrm{x}^{*}, \mathrm{y}-\mathrm{x}\right\rangle \leqslant 0, \forall \mathrm{y} \in \mathrm{C}\right\},
$$

for $x \in C$ and is empty otherwise. Then, $0 \in M x+N_{C}(x)$ if and only if $x \in C$ satisfies the variational inequalities of $\langle M x, y-x\rangle \geqslant 0$ for all $y \in C$.

Fixed point theory of nonexpansive mappings has been applied to zero point problem of accretive operators, see $[8,12,15,18]$ and the references therein. One of the most popular techniques for solving the zero point problem goes back to the work of Browder [6]. The basic idea is to reduce the zero point problem to a fixed point problem of operator $J_{r}^{A}:=(\operatorname{Id}+r A)^{-1}$, where $r$ is a positive real number and Id is the identity mapping, which is called the resolvent of $A$. Bruck [7] proposed a regularization iterative algorithm and proved the strong convergence of the iterative algorithm.

In this paper, we are interested in finding iteratively a common solution of a zero point problem of an accretive operator $A$ and a fixed point problem of a nonexpansive mapping $S$ via a viscosity approximation method involving a $\tau$-contractive mapping. Our results improve the corresponding results in $[8,15,17,18]$.

\section{Preliminaries}

Let $E$ be a Banach space and let $E^{*}$ be the dual space of $E$. Let $C$ be a closed convex subset $C$ of $E$. Recall that $C$ is said to have the normal structure if for each bounded closed convex subset $D$ of $C$ which contains at least two points, there exists an element $x$ of $D$ which is not a diametral point of $K$, i.e.,

$$
\operatorname{diam}(D)>\sup \{\|x-y\|: y \in D\},
$$

where $\operatorname{diam}(\mathrm{D})$ is the diameter of $\mathrm{D}$. Let $\langle\cdot, \cdot\rangle$ denote the pairing between $E$ and $E^{*}$. The normalized duality mapping $\mathrm{J}: \mathrm{E} \rightarrow 2^{\mathrm{E}^{*}}$ is defined by

$$
J(x)=\left\{f \in E^{*}:\langle x, f\rangle=\|x\|^{2}=\|f\|^{2}\right\}, \quad \forall x \in E .
$$

In the sequel, we use $j$ to denote the single-valued normalized duality mapping.

Let $S^{E}=\{x \in E:\|x\|=1\}$. Recall that $E$ is said to have a Gâteaux differentiable norm if the limit

$$
\lim _{t \rightarrow 0} \frac{\|x+t y\|-\|x\|}{t}
$$

exists for each $x, y \in S^{E}$. $E$ is said to be uniformly smooth or said to be have a uniformly Fréchet differentiable norm if the limit is attained uniformly for $x, y \in S^{E}$. E is said to have a uniformly Gâteaux differentiable norm if for each $y \in \mathrm{U}_{\mathrm{E}}$, the limit is attained uniformly for all $x \in S^{\mathrm{E}}$. It is known that if the norm of $E$ is uniformly Gâteaux differentiable, then duality mapping J is single-valued and uniformly norm to weak* continuous on each bounded subset of $E$.

Let Id denote the identity operator on $E$. An operator $A \subset E \times E$ with domain $\operatorname{Dom}(A)=\{z \in E$ : $A z \neq \emptyset\}$ and range $\operatorname{Ran}(A)=\cup\{A z: z \in D(A)\}$ is said to be accretive if for each $x_{i} \in D(A)$ and $y_{i} \in A x_{i}$, $i=1,2$, there exists $j\left(x_{1}-x_{2}\right) \in J\left(x_{1}-x_{2}\right)$ such that

$$
\left\langle y_{1}-y_{2}, j\left(x_{1}-x_{2}\right)\right\rangle \geqslant 0 .
$$

From Kato [13], we see that $A$ is accretive if and only if for all $\lambda>0,\left(x_{1}, y_{1}\right) \in A$ and $\left(x_{2}, y_{2}\right) \in A$, we have

$$
\left\|x_{1}-x_{2}\right\| \leqslant\left\|x_{1}-x_{2}+\lambda\left(y_{1}-y_{2}\right)\right\| .
$$

From the viewpoint of geometry, accretive operator $A \subset E \times E$ has the following properties: the range of accretive operator $I+\lambda A$ increases, that is, $I+\lambda A$ is expansive. An accretive operator $A$ is said to be 
$m$-accretive if $\operatorname{Ran}(I+r A)=E$ for all $r>0$. In a real Hilbert space, an operator $A$ is $m$-accretive if and only if $A$ is maximal monotone. In this paper, we use $A^{-1}(0)$ to denote the set of zeros of $A$. Interest in accretive operators stems mainly from their firm connection with equations of evolution, such as, heat, wave or Schrödinger equations.

For an accretive operator $A$, we can define a nonexpansive mapping $(I d+r A)^{-1}: \operatorname{Ran}(I+r A) \rightarrow$ $\operatorname{Dom}(A)$, which is called the resolvent of $A$.

Let $S: C \rightarrow C$ be a mapping and its fixed point set is denoted by $F(S)$. Recall that $S$ is said to be contractive if there exists a constant $\tau \in(0,1)$ such that

$$
\|S x-S y\| \leqslant \tau\|x-y\|, \quad \forall x, y \in C .
$$

We also call $S$ is a $\tau$-contraction. $S$ is said to be strongly pseudocontraction if there exist a constant $\tau \in(0,1)$ and some $j(x-y) \in J(x-y)$ such that

$$
\langle S x-S y, j(x-y)\rangle \leqslant \alpha\|x-y\|^{2}, \quad \forall x, y \in C .
$$

We also call $S$ is a $\tau$-strong pseudocontraction. $S$ is said to be nonexpansive if

$$
\|S x-S y\| \leqslant\|x-y\|, \quad \forall x, y \in C .
$$

One classical way to study nonexpansive mappings is to use contractions to approximate a nonexpansive mapping. Take $t \in(0,1)$ and define a contraction $S^{T, t}: C \rightarrow C$ by

$$
S^{T, t} x=t T+(1-t) S x, \quad \forall x \in C,
$$

where $T: C \rightarrow C$ is a $\tau$-contraction. Banach's contraction mapping principle guarantees that $S^{T, t}$ has a unique fixed point $x_{S, T, t}$ in $C$. That is,

$$
x_{S, T, t}=t T x_{S, T, t}+(1-t) S x_{S, T, t} .
$$

Moudafi [15] proved that $x_{S, T, t}$ converges strongly to a fixed point of $S$ in the framework of Banach space. For the results in the framework of Banach spaces, one refers to $[5,15,16,19]$ and the references therein.

Recently, Chang et al. [8] studied the following iterative scheme for accretive and nonexpansive operators via a viscosity approximate method:

$$
\left\{\begin{array}{l}
x_{0} \in C \\
y_{n}=\beta_{n} x_{n}+\left(1-\beta_{n}\right) S(I d+r A)^{-1} x_{n}, \\
x_{n+1}=\alpha_{n} f\left(x_{n}\right)+\left(1-\alpha_{n}\right) y_{n}, \quad \forall n \geqslant 0
\end{array}\right.
$$

where $r$ is a positive real number sequence, Id is the identity operator, $\left\{\alpha_{n}\right\}$ and $\left\{\beta_{n}\right\}$ are two real number sequences in $(0,1), f$ is a contraction, $S$ is a nonexpansive mapping and $A$ is an accretive operator. Under some suitable restrictions imposed on the above sequences, they obtained a strong convergence theorem of common solution to problems of $S x=x$ and $A x=0$.

Motivated by the above results, we investigate a zero point problem of an m-accretive operators and a fixed point problem of a nonexpansive mapping via a viscosity approximation method in a nonsmooth Banach space. We prove a strong convergence theorem of common solutions with mild restrictions imposed on the control sequences. It deserves to mention that control sequence $\left\{r_{n}\right\}$ is variable and the framework of the space is general in our convergence theorem comparing with Chang-Lee-Chan's results [8]. To prove our main results, we need the following tools.

Lemma 2.1 ([22]). Let $\left\{x_{n}\right\}$ and $\left\{y_{n}\right\}$ be bounded sequences in a Banach space $E$ and $\left\{\beta_{n}\right\}$ be a sequence in $[0,1]$ with

$$
0<\liminf _{n \rightarrow \infty} \beta_{n} \leqslant \limsup _{n \rightarrow \infty} \beta_{n}<1 .
$$


Suppose that

$$
x_{n+1}=\left(1-\beta_{n}\right) y_{n}+\beta_{n} x_{n}, \quad \forall n \geqslant 1,
$$

and

$$
\limsup _{n \rightarrow \infty}\left(\left\|y_{n+1}-y_{n}\right\|-\left\|x_{n+1}-x_{n}\right\|\right) \leqslant 0 .
$$

Then $\lim _{n \rightarrow \infty}\left\|y_{n}-x_{n}\right\|=0$.

Lemma 2.2 ([12]). In a Banach space $\mathrm{E}$, there holds the inequality

$$
\|x+y\|^{2} \leqslant\|x\|^{2}+2\langle y, j(x+y)\rangle, \quad \forall x, y \in E,
$$

where $j(x+y) \in J(x+y)$.

Lemma 2.3 ([18]). Let $\mathrm{E}$ be a real reflexive Banach space with the uniformly Gâteaux differentiable norm and $\mathrm{C}$ be a nonempty closed convex subset of $\mathrm{E}$ which has the normal structure. Let $\mathrm{S}: \mathrm{C} \rightarrow \mathrm{C}$ be a nonexpansive mapping with a fixed point and $\mathrm{T}: \mathrm{C} \rightarrow \mathrm{C}$ be a fixed contraction with the coefficient $\tau \in(0,1)$. Let $\left\{\mathrm{x}_{\mathrm{S}, \mathrm{T}, \mathrm{t}}\right\}$ be a sequence defined as follows

$$
\mathrm{x}_{\mathrm{S}, \mathrm{T}, \mathrm{t}}=\mathrm{tT} \mathrm{x}_{\mathrm{t}}+(1-\mathrm{t}) \mathrm{S} \mathrm{x}_{\mathrm{S}, \mathrm{T}, \mathrm{t}},
$$

where $t \in(0,1)$. Then $\left\{x_{\mathrm{t}}\right\}$ converges strongly as $\mathrm{t} \rightarrow 0$ to a fixed point $x^{*}$ of $\mathrm{S}$, which is the unique solution in $\mathrm{F}(\mathrm{S})$ to the following variational inequality

$$
\left\langle T x^{*}-x^{*}, j\left(x^{*}-p\right)\right\rangle \geqslant 0, \quad \forall p \in F(S) .
$$

Lemma 2.4 ([14]). Let $\left\{a_{n}\right\},\left\{b_{n}\right\}$ and $\left\{c_{n}\right\}$ be three nonnegative real sequences satisfying

$$
a_{n+1} \leqslant\left(1-t_{n}\right) a_{n}+b_{n}+c_{n}, \quad \forall n \geqslant 0,
$$

where $\left\{t_{n}\right\}$ is a sequence in $(0,1)$. Assume that the following conditions are satisfied

(a) $\sum_{n=0}^{\infty} t_{n}=\infty$ and $b_{n}=o\left(t_{n}\right)$;

(b) $\sum_{n=0}^{\infty} c_{n}<\infty$.

Then $\lim _{n \rightarrow \infty} a_{n}=0$.

Lemma 2.5 ([3]). Let $\mathrm{E}$ be a Banach space and $\mathrm{A}$ an m-accretive operator. For $\lambda>0$ and $\mu>0$ and $x \in \mathrm{E}$, we have

$$
\mathrm{J}_{\lambda} x=\mathrm{J}_{\mu}\left(\frac{\mu}{\lambda} x+\left(1-\frac{\mu}{\lambda}\right) \mathrm{J}_{\lambda} x\right),
$$

where $\mathrm{J}_{\lambda}=(\mathrm{I}+\lambda \mathrm{A})^{-1}$ and $\mathrm{J}_{\mu}=(\mathrm{I}+\mu \mathrm{A})^{-1}$.

\section{Main results}

Theorem 3.1. Let $\mathrm{E}$ be a real reflexive Banach space and let $\mathrm{A}$ be an $\mathrm{m}$-accretive operators in $\mathrm{E}$. Assume that $\mathrm{C}:=\overline{\operatorname{Dom}(\mathrm{A})}$ is convex and has the normal structure and $\mathrm{E}$ has a uniformly Gâteaux differentiable norm. Let $\mathrm{S}: \mathrm{C} \rightarrow \mathrm{C}$ be a nonexpansive mapping with a nonempty fixed point set and $\mathrm{T}: \mathrm{C} \rightarrow \mathrm{C}$ be a $\tau$-contractive mapping. Let $\left\{\alpha_{n}\right\}$ and $\left\{\beta_{n}\right\}$ be real number sequences in $(0,1)$. Let $\left\{x_{n}\right\}$ be a sequence generated in the following manner:

$$
\left\{\begin{array}{l}
x_{0} \in C \\
y_{n}=\beta_{n} S\left(I d+r_{n} A\right)^{-1}\left(e r_{n}+x_{n}\right)+\left(1-\beta_{n}\right) x_{n} \\
x_{n+1}=\alpha_{n} T x_{n}+\left(1-\alpha_{n}\right) y_{n}, \quad \forall n \geqslant 0
\end{array}\right.
$$

where $\left\{r_{n}\right\}$ is a positive real number sequence, $\left\{e_{n}\right\}$ is a bounded sequence in $\mathrm{E}$, and $\mathrm{Id}$ is the identity operator. Assume that the above control sequences satisfy the following restrictions: 
$\lim _{n \rightarrow \infty} \alpha_{n}=0, \sum_{n=1}^{\infty} \alpha_{n}=\infty, 0<\liminf _{n \rightarrow \infty} \beta_{n} \leqslant \limsup _{n \rightarrow \infty} \beta_{n}<1, \lim _{n \rightarrow \infty} r_{n}=r \in(0, \mathbb{R})^{+}$and $\mathrm{F}=\left(\mathrm{S}(\mathrm{Id}+\mathrm{rA})^{-1}\right)=\mathrm{A}^{-1}(0) \cap \mathrm{F}(\mathrm{S}) \neq \emptyset$.

Then $\left\{x_{n}\right\}$ converges strongly to a common solution $\bar{x}$ of problems $A x=0$ and $S x=x$. Furthermore, $\bar{x}$ is the unique solution of generality variational inequality

$$
\langle f(\bar{x})-\bar{x}, j(\bar{x}-y)\rangle \geqslant 0, \quad \forall y \in F(S) \cap A^{-1}(0) .
$$

Proof. We first show that $\left\{x_{n}\right\}$ and $\left\{y_{n}\right\}$ are bounded sequences in C. Fixing $p \in A^{-1}(0) \cap$ Fix $(S)$, we see that

$$
\begin{aligned}
\left\|y_{n}-p\right\| & =\left\|\beta_{n} S\left(\operatorname{Id}+r_{n} A\right)^{-1}\left(e r_{n}+x_{n}\right)+\left(1-\beta_{n}\right) x_{n}-p\right\| \\
& \leqslant \beta_{n}\left\|S\left(\operatorname{Id}+r_{n} A\right)^{-1}\left(e r_{n}+x_{n}\right)-S\left(\operatorname{Id}+r_{n} A\right)^{-1} p\right\|+\left(1-\beta_{n}\right)\left\|x_{n}-p\right\| \\
& \leqslant \beta_{n}\left\|\left(\operatorname{Id}+r_{n} A\right)^{-1}\left(e r_{n}+x_{n}\right)-\left(\operatorname{Id}+r_{n} A\right)^{-1} p\right\|+\left(1-\beta_{n}\right)\left\|x_{n}-p\right\| \\
& \leqslant \beta_{n}\left\|\left(e r_{n}+x_{n}\right)-p\right\|+\left(1-\beta_{n}\right)\left\|x_{n}-p\right\| \\
& \leqslant\left\|x_{n}-p\right\|+\left\|e r_{n}\right\| .
\end{aligned}
$$

Hence, we have

$$
\begin{aligned}
\left\|x_{n+1}-p\right\| & =\left\|\alpha_{n} T x_{n}+\left(1-\alpha_{n}\right) y_{n}-p\right\| \\
& \leqslant \alpha_{n}\left\|T x_{n}-p\right\|+\left(1-\alpha_{n}\right)\left\|y_{n}-p\right\| \\
& \leqslant \alpha_{n}\left\|T x_{n}-T p\right\|+\left(1-\alpha_{n}\right)\left\|y_{n}-p\right\|+\alpha_{n}\|T p-p\| \\
& \leqslant\left(1-\alpha_{n}(1-\tau)\right)\left\|x_{n}-p\right\|+\alpha_{n}(1-\tau) \frac{\|T p-p\|}{1-\tau}+\left(1-\alpha_{n}\right)\left\|e r_{n}\right\| \\
& \leqslant \max _{\{}\left\{\left\|x_{0}-p\right\|, \frac{\|T p-p\|}{1-\tau}\right\}+\left\|r_{n}\right\| .
\end{aligned}
$$

Since $\sum_{n=0}^{\infty} e r_{n}<\infty$, we find that $\left\{x_{n}\right\}$ is bounded, so is $\left\{y_{n}\right\}$. From Lemma 2.5, one has

$$
\begin{aligned}
&\left\|\left(I d+r_{n} A\right)^{-1}\left(e r_{n}+x_{n}\right)-\left(I d+r_{n+1} A\right)^{-1}\left(e r_{n+1}+x_{n+1}\right)\right\| \\
&= \|\left(I d+r_{n} A\right)^{-1}\left(e r_{n}+x_{n}\right)-\left(I d+r_{n} A\right)^{-1}\left(\frac{r_{n}}{r_{n+1}}\left(e r_{n+1}+x_{n+1}\right)\right. \\
&\left.+\left(1-\frac{r_{n}}{r_{n+1}}\right)\left(I d+r_{n+1} A\right)^{-1}\left(e r_{n+1}+x_{n+1}\right)\right) \| \\
& \leqslant \| \frac{r_{n}}{r_{n+1}}\left(e r_{n+1}+x_{n+1}\right) \\
&+\left(1-\frac{r_{n}}{r_{n+1}}\right)\left(I d+r_{n+1} A\right)^{-1}\left(e r_{n+1}+x_{n+1}\right)-\left(e r_{n}+x_{n}\right) \| \\
& \leqslant \| \frac{r_{n}}{r_{n+1}}\left(e r_{n+1}+x_{n+1}-e r_{n}-x_{n}\right) \\
&+\frac{r_{n+1}-r_{n}}{r_{n+1}}\left(\left(I d+r_{n+1} A\right)^{-1}\left(e r_{n+1}+x_{n+1}\right)-\left(e r_{n}+x_{n}\right)\right) \| \\
& \leqslant\left\|\left(e r_{n+1}+x_{n+1}-e r_{n}-x_{n}\right)+\frac{r_{n+1}-r_{n}}{r_{n+1}} \Omega_{n}\right\| \\
& \leqslant\left\|x_{n+1}-x_{n}\right\|+\frac{\left|r_{n+1}-r_{n}\right|}{r_{n+1}}\left\|\Omega_{n}\right\|+\left\|e r_{n+1}\right\|+\left\|e r_{n}\right\|,
\end{aligned}
$$

where $\Omega_{n}=\left(\operatorname{Id}+r_{n+1} A\right)^{-1}\left(e r_{n+1}+x_{n+1}\right)-e r_{n+1}-x_{n+1}$. Putting $z_{n}=\frac{x_{n+1}-\left(1-\beta_{n}\right) x_{n}}{\beta_{n}}$, we have

$$
\begin{aligned}
z_{n+1}-z_{n} & =\frac{x_{n+2}-\left(1-\beta_{n+1}\right) x_{n+1}}{\beta_{n+1}}-\frac{x_{n+1}-\left(1-\beta_{n}\right) x_{n}}{\beta_{n}} \\
& =\frac{\alpha_{n+1}\left(T x_{n+1}-y_{n+1}\right)+y_{n+1}-\left(1-\beta_{n+1}\right) x_{n+1}}{\beta_{n+1}}
\end{aligned}
$$




$$
\begin{aligned}
& -\frac{\alpha_{n}\left(T x_{n}-y_{n}\right)+y_{n}-\left(1-\beta_{n}\right) x_{n}}{\beta_{n}} \\
= & \frac{\alpha_{n+1}\left(T x_{n+1}-y_{n+1}\right)+\beta_{n+1} S\left(I d+r_{n+1} A\right)^{-1}\left(e r_{n+1}+x_{n+1}\right)}{\beta_{n+1}} \\
& -\frac{\alpha_{n}\left(T x_{n}-y_{n}\right)+\beta_{n} S\left(I d+r_{n} A\right)^{-1}\left(e r_{n}+x_{n}\right)}{\beta_{n}} \\
= & \alpha_{n+1} \frac{T x_{n+1}-y_{n+1}}{\beta_{n+1}}-\alpha_{n} \frac{T x_{n}-y_{n}}{\beta_{n}} \\
& +S\left(I d+r_{n+1} A\right)^{-1}\left(e r_{n+1}+x_{n+1}\right)-S\left(I d+r_{n} A\right)^{-1}\left(e r_{n}+x_{n}\right) .
\end{aligned}
$$

This in turn implies that

$$
\begin{aligned}
\left\|z_{n+1}-z_{n}\right\| \leqslant & \alpha_{n+1} \frac{\left\|T x_{n+1}-y_{n+1}\right\|}{\beta_{n+1}}+\alpha_{n} \frac{\left\|T x_{n}-y_{n}\right\|}{\beta_{n}} \\
& +\left\|S\left(\operatorname{Id}+r_{n+1} A\right)^{-1}\left(e r_{n+1}+x_{n+1}\right)-S\left(I d+r_{n} A\right)^{-1}\left(e r_{n}+x_{n}\right)\right\| \\
\leqslant & \alpha_{n+1} \frac{\left\|T x_{n+1}-y_{n+1}\right\|}{\beta_{n+1}}+\alpha_{n} \frac{\left\|T x_{n}-y_{n}\right\|}{\beta_{n}} \\
& +\left\|\left(I d+r_{n+1} A\right)^{-1}\left(e r_{n+1}+x_{n+1}\right)-\left(I d+r_{n} A\right)^{-1}\left(e r_{n}+x_{n}\right)\right\| .
\end{aligned}
$$

Combining (3.1) with (3.2), one sees that

$$
\begin{aligned}
\left\|z_{n+1}-z_{n}\right\|-\left\|x_{n+1}-x_{n}\right\| \leqslant & \alpha_{n+1} \frac{\left\|T x_{n+1}-y_{n+1}\right\|}{\beta_{n+1}}+\alpha_{n} \frac{\left\|T x_{n}-y_{n}\right\|}{\beta_{n}} \\
& +\frac{\left|r_{n+1}-r_{n}\right|}{r_{n+1}}\left\|\Omega_{n}\right\|+\left\|e r_{n+1}\right\|+\left\|e r_{n}\right\| .
\end{aligned}
$$

From the restrictions imposed on the control sequences, we have

$$
\limsup _{n \rightarrow \infty}\left(\left\|z_{n+1}-z_{n}\right\|-\left\|x_{n}-x_{n+1}\right\|\right) \leqslant 0 .
$$

By virtue of Lemma 2.1, we have

$$
\lim _{n \rightarrow \infty}\left\|z_{n}-x_{n}\right\|=0
$$

This implies that

$$
\lim _{n \rightarrow \infty}\left\|x_{n+1}-x_{n}\right\|=0
$$

and

$$
\lim _{n \rightarrow \infty}\left\|y_{n}-x_{n}\right\|=0 \text {. }
$$

In view of $S\left(\operatorname{Id}+r_{n} A\right)^{-1}\left(e r_{n}+x_{n}\right)-x_{n}=\frac{y_{n}-x_{n}}{\beta_{n}}$ and using the restriction imposed on $\left\{\beta_{n}\right\}$, one has

$$
\lim _{n \rightarrow \infty}\left\|S\left(\operatorname{Id}+r_{n} A\right)^{-1}\left(e r_{n}+x_{n}\right)-x_{n}\right\|=0 .
$$

Since

$$
\begin{aligned}
& \left\|S\left(\operatorname{Id}+r_{n} A\right)^{-1} x_{n}-x_{n}\right\| \\
& \quad \leqslant\left\|S\left(\operatorname{Id}+r_{n} A\right)^{-1} x_{n}-S\left(\operatorname{Id}+r_{n} A\right)^{-1}\left(e r_{n}+x_{n}\right)\right\|+\left\|S\left(\operatorname{Id}+r_{n} A\right)^{-1}\left(e r_{n}+x_{n}\right)-x_{n}\right\| \\
& \quad \leqslant\left\|\left(\operatorname{Id}+r_{n} A\right)^{-1} x_{n}-\left(\operatorname{Id}+r_{n} A\right)^{-1}\left(e r_{n}+x_{n}\right)\right\|+\left\|S\left(\operatorname{Id}+r_{n} A\right)^{-1}\left(e r_{n}+x_{n}\right)-x_{n}\right\| \\
& \quad \leqslant\left\|S\left(\operatorname{Id}+r_{n} A\right)^{-1}\left(e r_{n}+x_{n}\right)-x_{n}\right\|+\left\|e r_{n}\right\|,
\end{aligned}
$$


we find from (3.3) that

$$
\lim _{n \rightarrow \infty}\left\|S\left(\operatorname{Id}+r_{n} A\right)^{-1} \chi_{n}-x_{n}\right\|=0 .
$$

From Lemma 2.5, we obtain that

$$
\begin{aligned}
\left\|\left(I d+r_{n} A\right)^{-1}\left(x_{n}\right)-(I d+r A)^{-1}\left(x_{n}\right)\right\| \\
\quad=\left\|(I d+r A)^{-1}\left(\frac{r}{r_{n}} x_{n}+\left(1-\frac{r}{r_{n}}\right)\left(I d+r_{n} A\right)^{-1} x_{n}\right)-(I d+r A)^{-1} x_{n}\right\| \\
\quad \leqslant\left\|\left(\frac{r}{r_{n}} x_{n}+\left(1-\frac{r}{r_{n}}\right)\left(I d+r_{n} A\right)^{-1} x_{n}\right)-x_{n}\right\| \\
\quad \leqslant\left\|\left(1-\frac{r}{r_{n}}\right)\left(\left(I d+r_{n} A\right)^{-1} x_{n}-x_{n}\right)\right\| .
\end{aligned}
$$

It follows that

$$
\lim _{n \rightarrow \infty}\left\|\left(\operatorname{Id}+r_{n} A\right)^{-1}\left(x_{n}\right)-(I d+r A)^{-1}\left(x_{n}\right)\right\|=0 .
$$

Since

$$
\begin{aligned}
\| x_{n}- & S(\operatorname{Id}+r A)^{-1}\left(x_{n}\right) \| \\
& \leqslant\left\|x_{n}-S\left(\operatorname{Id}+r_{n} A\right)^{-1}\left(x_{n}\right)\right\|+\left\|S\left(\operatorname{Id}+r_{n} A\right)^{-1}\left(x_{n}\right)-S(\operatorname{Id}+r A)^{-1}\left(x_{n}\right)\right\| \\
& \leqslant\left\|x_{n}-S\left(\operatorname{Id}+r_{n} A\right)^{-1}\left(x_{n}\right)\right\|+\left\|\left(\operatorname{Id}+r_{n} A\right)^{-1}\left(x_{n}\right)-(\operatorname{Id}+r A)^{-1}\left(x_{n}\right)\right\|,
\end{aligned}
$$

we see from (3.4) and (3.5) that

$$
\lim _{n \rightarrow \infty}\left\|x_{n}-S(I d+r A)^{-1}\left(x_{n}\right)\right\|=0 .
$$

Since mapping $t f+(1-t) S(I d+r A)^{-1}$ is contractive, it has a unique fixed point. Next we use $x_{t}$ to denote the unique fixed point of $t f+(1-t) S(I d+r A)^{-1}$, that is,

$$
x_{t}=t f\left(x_{t}\right)+(1-t) S(I d+r A)^{-1} x_{t}, \quad \forall t \in(0,1) .
$$

From Lemma 2.3, we find that $x_{t} \rightarrow \bar{x}$, where $\bar{x}=\operatorname{Proj}_{F(S) \cap M^{-1}(0)} f(\bar{x})$, that is, $\bar{x}$ is the unique solution of generality variational inequality

$$
\langle f(\bar{x})-\bar{x}, j(\bar{x}-y)\rangle \geqslant 0, \quad \forall y \in F(S) \cap A^{-1}(0) .
$$

Next, we prove

$$
\limsup _{n \rightarrow \infty}\left\langle f(\bar{x})-\bar{x}, j\left(x_{n}-\bar{x}\right)\right\rangle \leqslant 0,
$$

For all $t \in(0,1)$, we see that

$$
\begin{aligned}
\left\|x_{t}-x_{n}\right\|^{2}= & (1-t)\left\langle S(I d+r A)^{-1} x_{t}-x_{n}, j\left(x_{t}-x_{n}\right)\right\rangle+t\left\langle f\left(x_{t}\right)-x_{n}, j\left(x_{t}-x_{n}\right)\right\rangle \\
= & (1-t)\left(\left\langle S(I d+r A)^{-1} x_{t}-S(I d+r A)^{-1} x_{n}, j\left(x_{t}-x_{n}\right)\right\rangle\right. \\
& \left.+\left\langle S(I d+r A)^{-1} x_{n}-x_{n}, j\left(x_{t}-x_{n}\right)\right\rangle\right) \\
& +t\left\langle f\left(x_{t}\right)-x_{t}, j\left(x_{t}-x_{n}\right)\right\rangle+t\left\langle x_{t}-x_{n}, j\left(x_{t}-x_{n}\right)\right\rangle \\
\leqslant & (1-t)\left(\left\|x_{t}-x_{n}\right\|^{2}+\left\|S(I d+r A)^{-1} x_{n}-x_{n}\right\|\left\|x_{t}-x_{n}\right\|\right) \\
& +t\left\langle f\left(x_{t}\right)-x_{t}, j\left(x_{t}-x_{n}\right)\right\rangle+t\left\|x_{t}-x_{n}\right\|^{2} \\
\leqslant & \left\|x_{t}-x_{n}\right\|^{2}+(1-t)\left\|S(I d+r A)^{-1} x_{n}-x_{n}\right\|\left\|x_{t}-x_{n}\right\|+t\left\langle f\left(x_{t}\right)-x_{t}, j\left(x_{t}-x_{n}\right)\right\rangle .
\end{aligned}
$$

It follows that

$$
\left\langle x_{t}-f\left(x_{t}\right), j\left(x_{t}-x_{n}\right)\right\rangle \leqslant \frac{1-t}{t}\left\|S(I d+r A)^{-1} x_{n}-x_{n}\right\|\left\|x_{t}-x_{n}\right\|, \quad \forall t \in(0,1) .
$$


It follows from (3.6) that

$$
\limsup _{n \rightarrow \infty}\left\langle x_{t}-f\left(x_{t}\right), j\left(x_{t}-x_{n}\right)\right\rangle \leqslant 0 .
$$

Since $x_{t} \rightarrow Q(u)$ as $t \rightarrow 0$ and the fact that $j$ is strong to weak* uniformly continuous on bounded subsets of $E$, we from (3.8) see that

$$
\limsup _{n \rightarrow \infty}\left\langle f(\bar{x})-\bar{x}, j\left(x_{n}-\bar{x}\right)\right\rangle \leqslant 0,
$$

that is, (3.7) holds.

Finally, we show that $x_{n} \rightarrow \bar{x}$ as $n \rightarrow \infty$. Using Lemma 2.2, we find that

$$
\begin{aligned}
\left.\| x_{n+1}-\bar{x}\right) \|^{2} & =\left\|\left(1-\alpha_{n}\right)\left(y_{n}-\bar{x}\right)+\alpha_{n}\left(f\left(x_{n}\right)-\bar{x}\right)\right\|^{2} \\
& \leqslant\left(1-\alpha_{n}\right)^{2}\left\|y_{n}-\bar{x}\right\|^{2}+2 \alpha_{n}\left\langle f\left(x_{n}\right)-\bar{x}, j\left(x_{n+1}-\bar{x}\right)\right\rangle \\
& \leqslant\left(1-\alpha_{n}\right)^{2}\left\|y_{n}-\bar{x}\right\|^{2}+2 \alpha_{n}\left\|f\left(x_{n}\right)-f(\bar{x})\right\|\left\|x_{n+1}-\bar{x}\right\|+2 \alpha_{n}\left\langle f(\bar{x})-\bar{x}, j\left(x_{n+1}-\bar{x}\right)\right\rangle \\
& \leqslant\left(1-\alpha_{n}\right)^{2}\left\|y_{n}-\bar{x}\right\|^{2}+2 \alpha_{n} \tau\left\|x_{n}-\bar{x}\right\|\left\|x_{n+1}-\bar{x}\right\|+2 \alpha_{n}\left\langle f(\bar{x})-\bar{x}, j\left(x_{n+1}-\bar{x}\right)\right\rangle .
\end{aligned}
$$

On the other hand, we have

$$
\begin{aligned}
\left\|y_{n}-\bar{x}\right\| & =\left\|\left(1-\beta_{n}\right)\left(x_{n}-\bar{x}\right)+\beta_{n}\left(S\left(\operatorname{Id}+r_{n} A\right)^{-1}\left(e r_{n}+x_{n}\right)-\bar{x}\right)\right\| \\
& \leqslant\left(1-\beta_{n}\right)\left\|x_{n}-\bar{x}\right\|+\beta_{n}\left\|S\left(\operatorname{Id}+r_{n} A\right)^{-1}\left(e r_{n}+x_{n}\right)-S\left(\operatorname{Id}+r_{n} A\right)^{-1} \bar{x}\right\| \\
& \leqslant\left(1-\beta_{n}\right)\left\|x_{n}-\bar{x}\right\|+\beta_{n}\left\|\left(\operatorname{Id}+r_{n} A\right)^{-1}\left(e r_{n}+x_{n}\right)-\left(\operatorname{Id}+r_{n} A\right)^{-1} \bar{x}\right\| \\
& \leqslant\left(1-\beta_{n}\right)\left\|x_{n}-\bar{x}\right\|+\beta_{n}\left\|\left(e r_{n}+x_{n}\right)-\bar{x}\right\| \\
& \leqslant\left\|x_{n}-\bar{x}\right\|+\left\|e r_{n}\right\| .
\end{aligned}
$$

Substituting (3.10) into (3.9), we find that

$$
\begin{aligned}
\left\|x_{n+1}-\bar{x}\right\|^{2} \leqslant & \left(1-\alpha_{n}\right)^{2}\left\|x_{n}-\bar{x}\right\|^{2}+\left(1-\alpha_{n}\right)^{2}\left\|e r_{n}\right\|\left(\left\|e r_{n}\right\|+2\left\|x_{n}-\bar{x}\right\|\right)+2 \alpha_{n} \tau\left\|x_{n}-\bar{x}\right\|\left\|x_{n+1}-\bar{x}\right\| \\
& +2 \alpha_{n}\left\langle f(\bar{x})-\bar{x}, j\left(x_{n+1}-\bar{x}\right)\right\rangle \\
\leqslant & \left(1-\alpha_{n}\right)^{2}\left\|x_{n}-\bar{x}\right\|^{2}+\left\|e r_{n}\right\|\left(\left\|e r_{n}\right\|+2\left\|x_{n}-\bar{x}\right\|\right)+\alpha_{n} \tau\left(\left\|x_{n}-\bar{x}\right\|^{2}+\left\|x_{n+1}-\bar{x}\right\|^{2}\right) \\
& +2 \alpha_{n}\left\langle f(\bar{x})-\bar{x}, j\left(x_{n+1}-\bar{x}\right)\right\rangle \\
\leqslant & \left(1-\alpha_{n}(2-\tau)+\alpha_{n}^{2}\right)\left\|x_{n}-\bar{x}\right\|^{2}+\left\|e r_{n}\right\|\left(\left\|e r_{n}\right\|+2\left\|x_{n}-\bar{x}\right\|\right)+\alpha_{n} \tau\left\|x_{n+1}-\bar{x}\right\|^{2} \\
& +2 \alpha_{n}\left\langle f(\bar{x})-\bar{x}, j\left(x_{n+1}-\bar{x}\right)\right\rangle .
\end{aligned}
$$

This implies that

$$
\begin{aligned}
\left\|x_{n+1}-\bar{x}\right\|^{2} \leqslant & \frac{1-\alpha_{n}(2-\tau)+\alpha_{n}^{2}}{1-\alpha_{n} \tau}\left\|x_{n}-\bar{x}\right\|^{2}+\frac{1}{1-\alpha_{n} \tau}\left\|e r_{n}\right\|\left(\left\|e r_{n}\right\|+2\left\|x_{n}-\bar{x}\right\|\right) \\
& +\frac{2 \alpha_{n}}{1-\alpha_{n} \tau}\left\langle f(\bar{x})-\bar{x}, j\left(x_{n+1}-\bar{x}\right)\right\rangle .
\end{aligned}
$$

Using Lemma 2.4, we can obtain the desired conclusion easily.

Remark 3.2. Theorem 3.1 improves the corresponding results in Chang et al. [8] in the following aspects.

(i) The framework of the space is extended to the case of nonsmooth Banach spaces.

(ii) Control sequence $\left\{r_{n}\right\}$ is variable with the iteration.

(iii) Control sequences $\left\{\alpha_{n}\right\}$ and $\left\{\beta_{n}\right\}$ are simpler.

From Theorem 3.1, we obtain the following result immediately. 
Corollary 3.3. Let $\mathrm{E}$ be a real reflexive Banach space and let $\mathrm{A}$ be an $\mathrm{m}$-accretive operators in $\mathrm{E}$. Assume that $\mathrm{C}:=\overline{\operatorname{Dom}(\mathrm{A})}$ is convex and has the normal structure and $\mathrm{E}$ has a uniformly Gâteaux differentiable norm. Let $\mathrm{T}: \mathrm{C} \rightarrow \mathrm{C}$ be a $\tau$-contractive mapping. Let $\left\{\alpha_{n}\right\}$ and $\left\{\beta_{n}\right\}$ be real number sequences in $(0,1)$. Let $\left\{x_{n}\right\}$ be a sequence generated in the following manner:

$$
\left\{\begin{array}{l}
x_{0} \in C \\
y_{n}=\beta_{n}\left(I d+r_{n} A\right)^{-1}\left(e r_{n}+x_{n}\right)+\left(1-\beta_{n}\right) x_{n}, \\
x_{n+1}=\alpha_{n} T x_{n}+\left(1-\alpha_{n}\right) y_{n}, \quad \forall n \geqslant 0
\end{array}\right.
$$

where $\left\{r_{n}\right\}$ is a positive real number sequence, $\left\{\boldsymbol{e r}_{\mathrm{n}}\right\}$ is a bounded sequence in $\mathrm{E}$, and $\mathrm{Id}$ is the identity operator. Assume that the above control sequences satisfy the following restrictions:

$\lim _{n \rightarrow \infty} \alpha_{n}=0, \sum_{n=1}^{\infty} \alpha_{n}=\infty, 0<\liminf _{n \rightarrow \infty} \beta_{n} \leqslant \limsup _{n \rightarrow \infty} \beta_{n}<1, \lim _{n \rightarrow \infty} r_{n}=r \in(0, \mathbb{R})^{+}$and $A^{-1}(0) \neq \emptyset$.

Then $\left\{x_{n}\right\}$ converges strongly to a common solution $\bar{x}$ of problems $A x=0$ and $S x=x$. Furthermore, $\bar{x}$ is the unique solution of generality variational inequality

$$
\langle f(\bar{x})-\bar{x}, j(\bar{x}-y)\rangle \geqslant 0, \quad \forall y \in A^{-1}(0) .
$$

Corollary 3.4. Let $\mathrm{E}$ be a real reflexive Banach space and let $\mathrm{A}$ be an $\mathrm{m}$-accretive operators in $\mathrm{E}$. Assume that $\mathrm{C}:=\overline{\operatorname{Dom}(\mathrm{A})}$ is convex and has the normal structure and $\mathrm{E}$ has a uniformly Gâteaux differentiable norm. Let $\mathrm{S}: \mathrm{C} \rightarrow \mathrm{C}$ be a nonexpansive mapping with a nonempty fixed point set. Let $\left\{\alpha_{n}\right\}$ and $\left\{\beta_{n}\right\}$ be real number sequences in $(0,1)$. Let $\left\{x_{n}\right\}$ be a sequence generated in the following manner:

$$
\left\{\begin{array}{l}
x_{0} \in C \\
y_{n}=\beta_{n} S\left(I d+r_{n} A\right)^{-1}\left(e r_{n}+x_{n}\right)+\left(1-\beta_{n}\right) x_{n} \\
x_{n+1}=\alpha_{n} x+\left(1-\alpha_{n}\right) y_{n}, \quad \forall n \geqslant 0
\end{array}\right.
$$

where $\mathrm{x}$ is a fixed element in $\mathrm{C},\left\{\mathrm{r}_{\mathrm{n}}\right\}$ is a positive real number sequence, $\left\{\mathrm{er}_{\mathrm{n}}\right\}$ is a bounded sequence in $\mathrm{E}$, and $\mathrm{Id}$ is the identity operator. Assume that the above control sequences satisfy the following restrictions:

$\lim _{n \rightarrow \infty} \alpha_{n}=0, \sum_{n=1}^{\infty} \alpha_{n}=\infty, 0<\liminf _{n \rightarrow \infty} \beta_{n} \leqslant \limsup _{n \rightarrow \infty} \beta_{n}<1, \lim _{n \rightarrow \infty} r_{n}=r \in(0, \mathbb{R})^{+}$and $\mathrm{F}=\left(\mathrm{S}(\mathrm{Id}+\mathrm{rA})^{-1}\right)=\mathrm{A}^{-1}(0) \cap \mathrm{F}(\mathrm{S}) \neq \emptyset$.

Then $\left\{x_{n}\right\}$ converges strongly to a common solution $\bar{x}$ of problems $A x=0$ and $S x=x$. Furthermore, $\bar{x}$ is the unique solution of generality variational inequality

$$
\langle x-\bar{x}, j(\bar{x}-y)\rangle \geqslant 0, \quad \forall y \in F(S) \cap A^{-1}(0) .
$$

\section{References}

[1] I. K. Argyros, S. George, Iterative regularization methods for nonlinear ill-posed operator equations with m-accretive mappings in Banach spaces, Acta Math. Sci. Ser. B Engl. Ed., 35 (2015), 1318-1324. 1

[2] I. K. Argyros, S. George, Extending the applicability of a new Newton-like method for nonlinear equations, Commun. Optim. Theory, 2016 (2016), 9 pages. 1

[3] V. Barbu, Nonlinear semigroups and differential equations in Banach spaces, Translated from the Romanian, Editura Academiei Republicii Socialiste România, Bucharest; Noordhoff International Publishing, Leiden, (1976). 2.5

[4] B. A. Bin Dehaish, A. Latif, H. O. Bakodah, X.-L. Qin, A regularization projection algorithm for various problems with nonlinear mappings in Hilbert spaces, J. Inequal. Appl., 2015 (2015), 14 pages. 1

[5] B. A. Bin Dehaish, X.-L. Qin, A. Latif, H. O. Bakodah, Weak and strong convergence of algorithms for the sum of two accretive operators with applications, J. Nonlinear Convex Anal., 16 (2015), 1321-1336. 2

[6] F. E. Browder, Existence and approximation of solutions of nonlinear variational inequalities, Proc. Nat. Acad. Sci. U.S.A., 56 (1966), 1080-1086. 1

[7] R. E. Bruck, Jr., A strongly convergent iterative solution of $0 \in \mathrm{U}(\mathrm{x})$ for a maximal monotone operator $\mathrm{U}$ in Hilbert space, J. Math. Anal. Appl., 48 (1974), 114-126. 1

[8] S.-S. Chang, H. W. J. Lee, C. K. Chan, Strong convergence theorems by viscosity approximation methods for accretive mappings and nonexpansive mappings, J. Appl. Math. Inform., 27 (2009), 59-68. 1, 2, 3.2 
[9] C. E. Chidume, Iterative solutions of nonlinear equations in smooth Banach spaces, Nonlinear Anal., 26 (1996), 18231834. 1

[10] S. Y. Cho, B. A. Bin Dehaish, X.-L. Qin, Weak convergence of a splitting algorithm in Hilbert spaces, J. Appl. Anal. Comput., 7 (2017), 427-438. 1

[11] S. Y. Cho, X.-L. Qin, L. Wang, Strong convergence of a splitting algorithm for treating monotone operators, Fixed Point Theory Appl., 2014 (2014), 15 pages. 1

[12] J. S. Jung, Y. J. Cho, H.-Y. Zhou, Iterative processes with mixed errors for nonlinear equations with perturbed m-accretive operators in Banach spaces, Appl. Math. Comput., 133 (2002), 389-406. 1, 2.2

[13] T. Kato, Nonlinear semigroups and evolution equations, J. Math. Soc. Japan, 19 (1967), 508-520. 2

[14] L. S. Liu, Ishikawa and Mann iterative process with errors for nonlinear strongly accretive mappings in Banach spaces, J. Math. Anal. Appl., 194 (1995), 114-125. 2.4

[15] A. Moudafi, Viscosity approximation methods for fixed-points problems, J. Math. Anal. Appl., 241 (2000), 46-55. 1, 2

[16] X.-L. Qin, S. Y. Cho, Convergence analysis of a monotone projection algorithm in reflexive Banach spaces, Acta Math. Sci. Ser. B Engl. Ed., 37 (2017), 488-502. 2

[17] X.-L. Qin, S. Y. Cho, J. K. Kim, On the weak convergence of iterative sequences for generalized equilibrium problems and strictly pseudocontractive mappings, Optimization, 61 (2012), 805-821. 1

[18] X.-L. Qin, S. Y. Cho, L. Wang, Iterative algorithms with errors for zero points of m-accretive operators, Fixed Point Theory Appl., 2013 (2013), 10 pages. 1, 2.3

[19] X.-L. Qin, J.-C. Yao, Weak convergence of a Mann-like algorithm for nonexpansive and accretive operators, J. Inequal. Appl., 2016 (2016), 9 pages. 2

[20] S. Reich, On fixed point theorems obtained from existence theorems for differential equations, J. Math. Anal. Appl., 54 (1976), 26-36. 1

[21] R. T. Rockafellar, Monotone operators and the proximal point algorithm, SIAM J. Control Optim., 14 (1976), 877-898. 1

[22] T. Suzuki, Strong convergence theorems for infinite families of nonexpansive mappings in general Banach spaces, Fixed Point Theory Appl., 2005 (2005), 21 pages. 1, 2.1

[23] H.-Y. Zhou, A characteristic condition for convergence of steepest descent approximation to accretive operator equations, J. Math. Anal. Appl., 271 (2002), 1-6. 1

[24] H.-Y. Zhou, Strong convergence of an explicit iterative algorithm for continuous pseudo-contractions in Banach spaces, Nonlinear Anal., 70 (2009), 4039-4046. 1 